\title{
Measurement of the Electromagnetic Field Backscattered by a Fractal Surface for the Verification of Electromagnetic Scattering Models
}

\author{
Giuseppe Ruello, Member, IEEE, Pablo Blanco-Sánchez, Antonio Iodice, Senior Member, IEEE, \\ Jordi J. Mallorquí, Member, IEEE, Daniele Riccio, Senior Member, IEEE, \\ Antoni Broquetas, Member, IEEE, and Giorgio Franceschetti, Life Fellow, IEEE
}

\begin{abstract}
Fractal geometry is widely accepted as an efficient theory for the characterization of natural surfaces; the opportunity of describing irregularity of natural surfaces in terms of few fractal parameters makes its use in direct and inverse electromagnetic (EM) scattering theories highly desirable. In this paper, we present an innovative procedure for manufacturing fractal surfaces and for measuring their scattering properties. A cardboard-aluminum fractal surface was built as a representation of a Weiestrass-Mandelbrot fractal process; the EM field scattered from it was measured in an anechoic chamber. A monostatic radarlike configuration was employed. Measurement results were compared to Kirchhoff approximation and small perturbation method closed-form results that were analytically obtained by employing the fractional Brownian motion to model the surface shape. Matching and discrepancies between theories and measurements are then discussed. Finally, fractal and classical surface models are compared as far as their use in the EM scattering is concerned.
\end{abstract}

Index Terms-Electromagnetic scattering by rough surfaces, fractals.

\section{INTRODUCTION}

A RELIABLE theory on the electromagnetic (EM) scattering from natural surfaces requires an efficient and accurate quantitative description of the natural surface shapes. The use of fractal geometry for this purpose is strongly suggested because it accounts for the complex behavior of nature by means of simple models.

Elegant methods for the evaluation of the EM field scattered from fractal surfaces were recently developed with encouraging results [1], [2]. So far, no scattering measurement campaigns on surfaces with known fractal parameters were carried out. Most of the measurement campaigns presented in the literature

Manuscript received September 5, 2007; revised June 19, 2008, December 12, 2008, and July 3, 2009. First published December 22, 2009; current version published March 24, 2010. This work was supported by the Spanish MCYT and EU FEDER funds under Project TEC2008-06764-C02-01.

G. Ruello, A. Iodice, D. Riccio, and G. Franceschetti are with the Department of Electronic and Telecommunication Engineering, University of Naples "Federico II," 80125 Naples, Italy (e-mail: ruello@unina.it; iodice@unina.it; Daniele.Riccio@unina.it; gfrance@unina.it).

P. Blanco-Sánchez, J. J. Mallorquí, and A. Broquetas are with the Department of Signal Theory and Communications, School of Telecommunication Engineering, Universitat Politecnica de Catalunya, 08034 Barcelona, Spain (e-mail: pblanco@tsc.upc.edu; mallorqui@tsc.upc.edu; toni@tsc.upc.edu).

Color versions of one or more of the figures in this paper are available online at http://ieeexplore.ieee.org.

Digital Object Identifier 10.1109/TGRS.2009.2036007 were performed on artificially constructed Gaussian surfaces [3]-[5] or on natural surfaces described by means of nonfractal parameters like the correlation length and standard deviation [1]. However, already in the 1970s, it was known that classical Gaussian parameters estimated from samples of natural surfaces depend on the size of the considered surfaces [6], [7]; this is considered as a major motivation for conceiving more appropriate models to describe natural surfaces. In 1973, Beckmann [6] expressed the need of a non-Gaussian description of natural surfaces for electromagnetic scattering evaluation purposes. $\mathrm{He}$ pointed out that the probability density function (pdf) of a natural surface can also be described by a Gaussian model, but the pdf could be noncritical for its scattering properties. In 1988, Wu et al. [8] agreed that non-Gaussian processes would be necessary for natural surface description. The introduction of the fractal geometry provided a powerful instrument for comprehending and quantitatively describing the irregular shapes of nature. As a matter of fact, fractal models account for the nonstationary and self-affine characteristics of actual processes, so that in the last decades, several researchers intensively used a fractal representation of natural surfaces (e.g., [1], [9]-[17] are a small but significant part of the huge literature on the topic). The use of fractals in remote sensing is highly desirable, also because the natural surfaces can be described by employing just few parameters, and this is very attractive for model-inversion purposes [18], [19].

In this paper, we present an innovative measurement campaign in a controlled environment carried out to test, in monostatic configuration, the theories on the electromagnetic scattering from fractal surfaces.

In Section II, we introduce the fundamentals of fractal models, and we recall the main concepts which inspired the surface construction. In particular, we define the fractional Brownian motion $(\mathrm{fBm})$, an everywhere continuous but nowhere differentiable process, which is the most suitable model to describe natural surfaces, and it is currently used in EM scattering theories. Then, in order to synthesize an fBm fractal surface, we introduce the Weierstrass-Mandelbrot function (WM) whose spectrum provides a reliable approximation of an $\mathrm{fBm}$ process spectrum. The values of the fractal parameters chosen for the numerical synthesis of the surface are consistent with typical natural surfaces. Then, we present the procedure to build the surface as a superposition of a cardboard structure, representing the low frequencies, and wrinkled aluminum foils, accounting for the high-frequency surface components. Employed 
materials guaranteed to produce a cost-effective, portable, and reliable fractal surface. The correspondence between synthesized and built-surface fractal parameters was checked by means of appropriate optical measurements [20].

In Section III, we recall how the fBm surface description can be used in conjunction with the Kirchhoff approximation (KA) and the small perturbation method (SPM) [1], in order to provide the second-order statistics of the scattered field [21], [22]. The choice of the KA and the SPM is related to the fact that these are the most used techniques in remote-sensing applications: from SAR data interpretation [23] to retrieving algorithms [18], [19].

Section IV is devoted to describe the procedure and the implementation of the EM scattering measurements, performed in the anechoic chamber of the Universitat Politècnica de Catalunya (UPC). A couple of X-band horn antennas was used to illuminate the built surface and measure the field backscattered from it. The built surface was mounted on a rotor, whose movements allowed the acquiring of a sufficient number of samples at different incidence angles (incidence angles in the range of $0^{\circ}-70^{\circ}$ were explored). Both horizontal- and verticalpolarized scattered fields were measured. The calibration procedure performed with the use of a trihedral corner reflector is also presented.

In Section V, we show the obtained results. Calibrated measured data are presented as a function of the incidence angle; they are then compared with the theoretical results in Section III. Matching and differences are deeply discussed. In addition, a discussion on the main advantages of fractals with respect to classical models in the EM scattering methods is presented.

The aim of Section VI is to summarize the rationale, approach, implementation, and results related with the presented work.

\section{Surface Construction}

In this section, we recall the basic principles of the fractal geometry, and we provide the rationale for the synthesis, the building, and the validation of the fractal surface employed in our experiments. A detailed description of these topics is provided in [20].

\section{A. Fractal Geometry}

The $\mathrm{fBm}$ is widely recognized as the most suitable fractal process to model natural surfaces. It allows the describing of the surface shape in terms of only two parameters, the dimensionless Hurst coefficient, $H$, and the incremental standard deviation at unitary distance, $s$, measured in $\mathrm{m}^{(1-H)}$. An $\mathrm{fBm}$ process represents a stochastic surface $z(x, y)$ if, for every $x, y$, $x^{\prime}$, and $y^{\prime}$, the pdf of its increments is Gaussian with zero mean and standard deviation $s \tau^{H}$

$$
\begin{aligned}
\operatorname{Pr}\left\{z(x, y)-z\left(x^{\prime}, y^{\prime}\right)<\bar{\zeta}\right\} & \\
= & \frac{1}{\sqrt{2 \pi} s \tau^{H}} \int_{-\infty}^{\bar{\zeta}} \exp \left(-\frac{\zeta^{2}}{2 s^{2} \tau^{2 H}}\right) d \zeta
\end{aligned}
$$

It can be demonstrated [13], [24] that a process satisfying (1) exists if $0<H<1$, and that (with probability one) any $\mathrm{fBm}$ sample surface has a fractal dimension $D=3-H$. Furthermore, if we consider the increments at distance $\tau=1 \mathrm{~m}$, their standard deviation is $s$. Therefore, the $s$ parameter represents an index of roughness. The higher its value, the rougher the surface [1].

Evaluation of the fBm power spectrum deserves special care due to the nonstationarity of the surface. Space-frequency and scale-frequency approaches are required in order to express the power spectral density of the surface. Both these approaches lead to a power-law spectrum [1], [13]

$$
W(k)=S_{0} k^{-\alpha}
$$

where $S_{0}$ and $\alpha$ are parameters depending on $H$ and $s[1]$ and $k$ is the spatial wavenumber.

The $\mathrm{fBm}$ is a regular stochastic process, whose synthesis is not a straightforward task, and it is usually accomplished in terms of appropriate functions, as detailed in the following section.

\section{B. Synthesis}

The synthesis of $\mathrm{fBm}$ processes can be obtained via displaced interpolation [25], spectral synthesis [1], wavelet [25] methods, and so on. In this paper, we use a WM function, which is a predictable process that allows an easy and controllable modeling of deterministic and stochastic processes as a function of few physical parameters.

A mathematical WM is a superposition of infinite sinusoidal tones with periods spaced by an irrational factor. Natural surfaces exhibit a fractal behavior in a wide but finite range of scales, so a physical WM [1] can be obtained by using a 2-D band-limited (i.e., with a finite number of tones, $M$ ) WM function $f(x, y)$

$$
f(x, y)=B \sum_{n=0}^{M-1} C_{n} \nu^{-H n} \sin \left(k_{0} \nu^{n}\left(x \cos \psi_{n}+y \sin \psi_{n}\right)+\phi_{n}\right)
$$

where $B$ is an amplitude scaling factor; $\nu$ is the irrational frequency scaling factor; $C_{n}, \psi_{n}$, and $\phi_{n}$ are random variables, accounting for amplitude, direction, and phase behavior of each tone, respectively [1]; and $k_{0}$ is the fundamental tone wavenumber. It was shown that the WM can be considered as a spectral sampled version of an $\mathrm{fBm}$ process with the same fractal dimension and with $S_{0}$ related to the WM parameters by [1]

$$
B^{2}=\frac{S_{0}}{2 \pi H} k_{0}^{-2 H}\left(\nu^{H}-\nu^{-H}\right) .
$$

The synthesis of a single surface calls for the setting of five parameters: $H, B, k_{0}, k_{M-1}$, and $\nu$. For the surface considered in this paper, their values were chosen according to the following rationale.

1) The $H$ value was set at 0.7 , because natural surfaces typically hold $H$ values ranging from 0.6 to 0.9 [24].

2) The $B$ value was set at $0.011 \mathrm{~m}$, in accordance to typical values for natural surfaces. This choice corresponds to an $s$ value of $0.063 \mathrm{~m}^{1-H}$, in accordance with typical natural values [24]. 
TABLE I

Simulated Surface Parameters

\begin{tabular}{c|c|c|c|c}
\hline $\begin{array}{c}k_{0} \\
{\left[\mathrm{~m}^{-1}\right]}\end{array}$ & $\begin{array}{c}B \\
{[\mathrm{~m}]}\end{array}$ & $H$ & $v$ & $\begin{array}{c}k_{M-1} \\
{\left[\mathrm{~m}^{-1}\right]}\end{array}$ \\
\hline 5.71 & 0.011 & 0.7 & $0.5 \mathrm{e}$ & 1943.8 \\
\hline \hline
\end{tabular}

3) The lowest roughness scale $k_{0}$ that contributes to the scattered field formation depends on the illuminated area and the anechoic chamber dimensions. In this paper, the fundamental tone wavenumber is of $5.71 \mathrm{~m}^{-1}$.

4) The highest roughness scale $k_{M-1}$ that contributes to the scattered field formation depends on the incident wavelength $\lambda$. Scales lower than a fraction of the wavelength do not contribute to the field formation. We fixed $\lambda=$ $3 \mathrm{~cm}$ as a reference wavelength, so that reliable measurements could be performed at frequencies included in the X-band. Therefore, $k_{M-1}=1943.8 \mathrm{~m}^{-1}$, that corresponds to a surface tone wavelength of $3.2 \mathrm{~mm}$, which is about $\lambda / 10$.

5) The $\nu$ value was set at $0.5 e$, as a tradeoff between the $\mathrm{fBm}$ sampling rate and the memory constraints. Such a choice leads to a number of tones of $M=20$ [20].

In Table I, the chosen numerical values are summarized, and a representation of the synthesized surface is shown in Fig. 1(a).

\section{Manufacturing}

The surface was built according to a two-step approach. The largest spatial scale roughness (from meters till $0.5 \mathrm{~cm}$ ) was assembled as superposition of cardboard layers shaped according to the synthesized surface-level curves with a step of $0.5 \mathrm{~cm}$ (i.e., $\lambda / 6$ ) [see Fig. 1(b)]. The choice of cardboard allowed an easy manufacturing and portability of the surface.

Once such a $1.5 \mathrm{~m} \times 1.5 \mathrm{~m}$ (i.e., $50 \lambda \times 50 \lambda$ ) macroscopic structure was built, we added the microscopic roughness by superposing two layers of aluminum foils [see Fig. 1(c)]. The first layer was glued directly to the cardboard without corrugation. The second was manually wrinkled and glued on the top. The intensity of the corrugations was not precontrolled but evaluated via laser scansion. Such a procedure allowed the considering of the built surface as perfect reflector. In Fig. 1(d), we show a top view of the built surface. Note that the aluminum foils cover up the seams, avoiding the possibility that they could bias the results with their nonfractal straight edges. The chosen surface shape is circular, with a $1.5-\mathrm{m}$ diameter, in order to minimize the border effects [20].

\section{Validation}

A high-precision laser (with resolution of $0.7 \mathrm{~mm}$ ) was employed to provide an accurate analysis of the built-surface properties. The measured data were processed in both the spatial and spectral domains, leading to the result that the fractal surface holds the prescribed synthesized parameters [20] within the range of scales of interest for electromagnetic scattering experiments [1]. The obtained measurements showed that the aluminum layers did not significantly change the surface roughness at the scales of interest for the electromagnetic scattering.

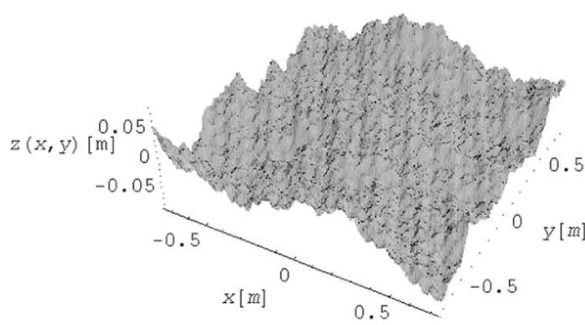

(a)

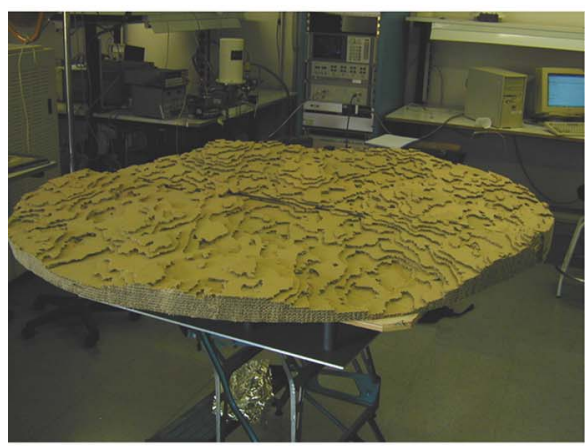

(b)

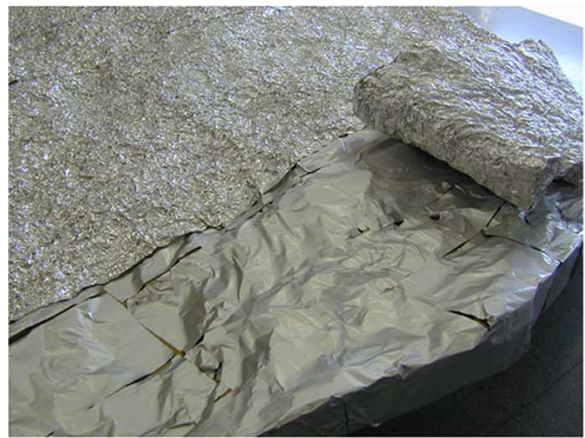

(c)

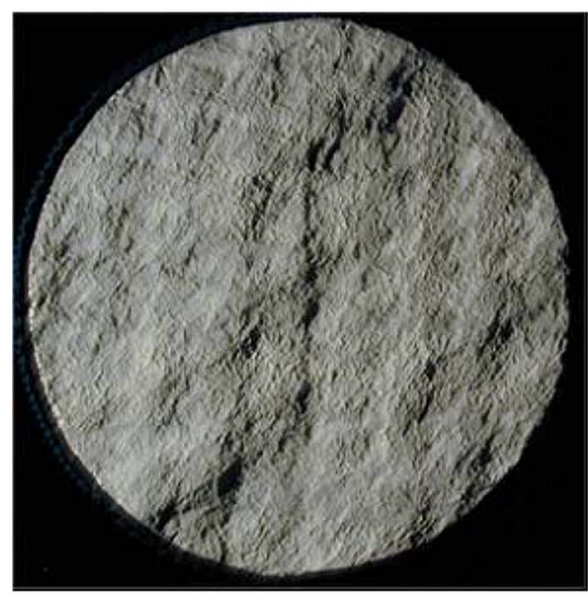

(d)

Fig. 1. (a) Synthesized surface. (b) Cardboard topography. (c) Detail on the aluminum layers. (d) Top view of the built surface.

\section{Electromagnetic Methods}

In this paper, we focus our attention on methods that provide simple and effective closed-form solutions, because their use is of interest in remote sensing applications [1], [18], [19], [26]. In particular, we focus our analysis on the mostly used electromagnetic methods, the KA and the SPM. These methods are intensively used in remote sensing applications for developing 
SAR interpretation tools [23] as well as inverse methods for retrieving physical parameters in remote sensing applications [18], [19].

The introduction of the $\mathrm{fBm}$ fractal process for surface profile description purposes allowed the development of improved versions of these electromagnetic scattering methods [1], [2], [21], [22], whose results are recalled in the following.

\section{A. KA Formulation}

The PO solution of the KA leads to express the scattered power density $S_{i}$ as a function of the surface parameters [1], [2]

$$
S_{i} \propto \int_{0}^{\infty} J_{0}\left(\eta_{x y} \tau\right) \exp \left(-\frac{1}{2} \eta_{z}^{2} s^{2} \tau^{2 H}\right) \tau d \tau
$$

where $J_{0}$ is the zeroth-order Bessel function and $\boldsymbol{\eta}=\mathbf{k}_{\mathbf{i}}-\mathbf{k}_{\mathbf{s}}=\left(\eta_{x}, \eta_{y}, \eta_{z}\right)$, where $\mathbf{k}_{\mathbf{i}}$ and $\mathbf{k}_{\mathbf{s}}$ are the incident and scattered wave vectors, respectively, $\eta_{x y}=\sqrt{\eta_{x}^{2}+\eta_{y}^{2}}$. The surface roughness, expressed in terms of the fractal parameters $s$ and $H$, as well as the incidence angle are accounted for in the arguments of the Bessel and the exponential functions. A complete treatise of the integral evaluation is well beyond the goals of this paper. In literature, closed-form solutions can be obtained via asymptotic expansions, as detailed in [1], [2].

\section{B. SPM Formulation}

The SPM formulation provides the radar cross section as a function of the surface spectrum. If we use the fBm spectrum in the expression of the first-order SPM radar cross section, we obtain the radar cross section as a function of the spectral surface parameters [1], [2], [22]

$$
\sigma_{p p}^{o}=4 k^{4} \cos ^{4} \vartheta\left|\beta_{p p}\right|^{2} \frac{S_{0}}{\pi(2 k \sin \vartheta)^{\alpha}}
$$

with $\beta_{p p}$ taking into account the polarization issue, and $S_{0}$ and $\alpha$ are the fBm power-law spectrum parameters [1], [2].

\section{Validity Limits}

The definition of the validity limits for electromagnetic scattering from fractal surfaces is still an open problem. This is mainly due to the fact that the $\mathrm{fBm}$ is not differentiable, and its use in the scattering evaluation requires a physical-based bandlimiting procedure. Such an operation influences the definition of the validity limits. So far, the validity limits are evaluated via relationships between fractal and classical parameters, as reported in [1], [2]. However, the equivalence between classical and fractal parameters requires subtle theoretical issues. To the best of our knowledge, no conclusive results exist in the available literature. In a recent paper [26], an empirical condition was defined in terms of the significant slope $s_{s}$ (the ratio between the root mean square (RMS) surface height and the wavelength of the dominant spectral peak). According to [26], for near-nadir incidence (incidence angles lower than $30^{\circ}$ ), the PO approach is applicable if $s_{s}<0.037 \cos ^{3} \theta$. For the surface employed in our experiment, the condition holds for every incidence angle less than $30^{\circ}$. Despite the fact that it does
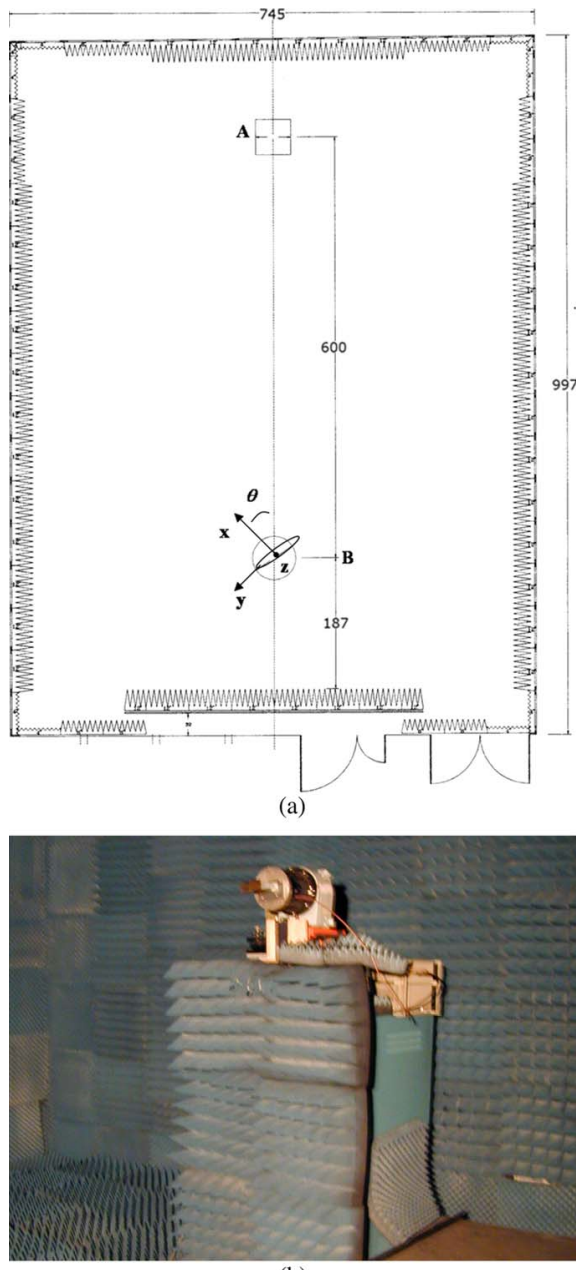

(b)

Fig. 2. (a) Top view of the anechoic chamber. A identifies the antenna position, B identifies the surface position. Linear dimensions are expressed in centimeters. (b) Image of the transmitting and receiving antennas.

not constitute a conclusive proof, this result suggests that, at least for near-nadir angles, validity limits are nearly fulfilled. A complete discussion on this topic requires a reformulation of the validity limits in terms of fractal parameters, but it goes beyond the goals of this paper and it is demanded to a future discussion.

\section{Electromagnetic Measurement Setup}

\section{A. Geometry}

The fractal surface, built in accordance with the procedure of Section II, was used to validate the EM scattering methods presented in Section III. In this section, we present the procedure to measure the EM field backscattered from this surface. The experiments were performed in the anechoic chamber at UPC. A top view of the measurement geometry is shown in Fig. 2(a).

Two horn antennas, one for transmitting and one for receiving [see Fig. 2(b)], were placed in a fixed position [see the A point in Fig. 2(a)]. The surface was mounted in position B [see Fig. 2(a)] at a distance of $5.75 \mathrm{~m}$ from the antennas, on a rollover azimuth positioning system that allows rotations both in 


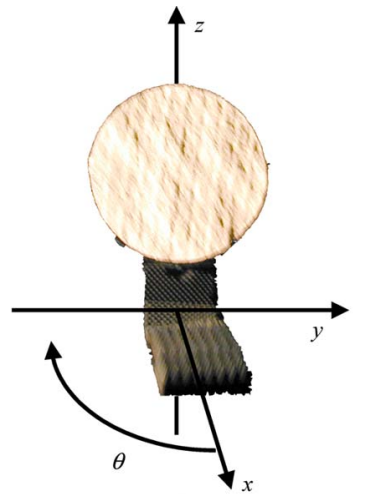

(a)

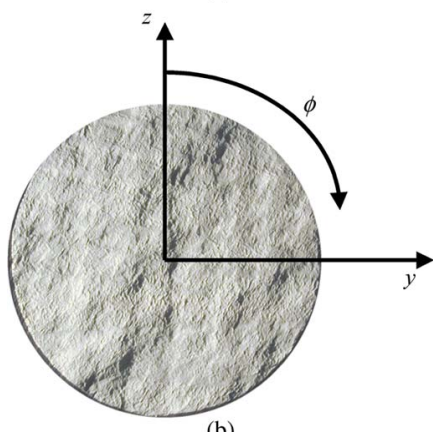

(b)

Fig. 3. Measurement geometry. The rotor allows the built-surface rotation around the (a) $z$ - and (b) $x$-axes.

the $(y, z)$ plane around the $x$-axis and in the $(x, y)$ plane along the $z$-axis, in accordance with the geometry of Fig. 3. The spot size of the illuminating beam covers the whole surface.

The rotations around the $z$-axis allowed the acquisition of scattered field samples at different incidence angles $\theta$ [see Figs. 2 and 3(a)]. At a given incidence angle, the rotations around the $x$-axis guarantee the acquisition of many independent scattered field samples.

\section{B. Measurement Procedure}

For each surface $(\theta, \phi)$ position, the X-band antennas acquired 401 samples, uniformly spaced within the frequency range from 7 to $12 \mathrm{GHz}$. Appropriate transmitting and receiving antenna rotations provided the acquisition of $\mathrm{HH}$ - and $\mathrm{VV}$ polarized fields.

The field backscattered from the fractal surface was measured using an HP8510 network analyzer for $\theta$ ranging from $0^{\circ}$ to $70^{\circ}$ with a $2^{\circ}$ step. For each $\theta$ value, 72 independent field values were acquired by rotating the surface with $\phi$ steps of $5^{\circ}$. In fact, the fractal surface under analysis is a single realization of an $\mathrm{fBm}$ stochastic process. It can be seen as a cell composed of multiple scatterers. By changing the illumination angle, the relative distribution of the scatterers changes, so that two acquisitions are different. The rotation of a resolution element by the angle $\phi$ moves the scattering centers. The range component of the displacement causes a slightly different phase shift for each scattering center, resulting in signal decorrelation. We choose a $\phi$ step of $5^{\circ}$, in accordance with the results found in the literature concerning the signal decorrelation for interferometric applications [27], [28]. In [27], the correlation coefficient

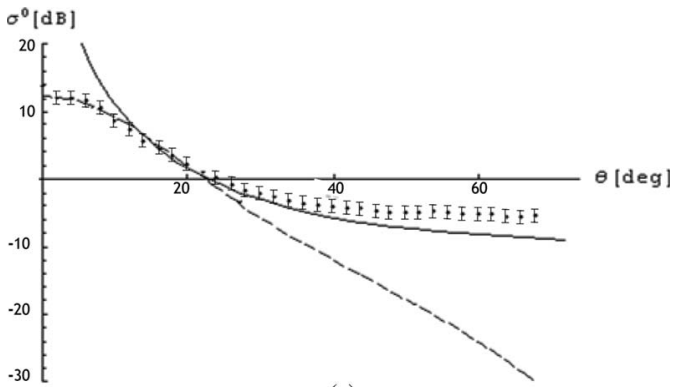

(a)

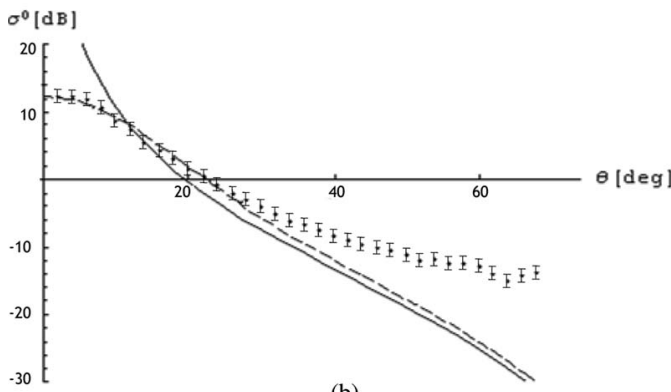

(b)

Fig. 4. Comparison between (dots) data, (dashed line) KA, and (solid line) SPM for (a) VV and (b) HH polarizations.

between the field scattered by a surface and the field scattered by the same surface rotated by an angle $\phi$ is computed as

$$
\rho_{\phi}= \begin{cases}1-\frac{2(\sin \theta)|\phi| r}{\lambda}, & \text { if } \frac{2(\sin \theta)|\phi| r}{\lambda}<1 \\ 0, & \text { otherwise }\end{cases}
$$

where $r$ is the antenna-surface distance. In fact, by using in this expression $\phi=5^{\circ}$, considering the values of $\lambda$ and $\theta$ belonging to the intervals defined earlier and considering $r=5.75 \mathrm{~m}$ (see Fig. 2), we obtain a null value of the correlation coefficient.

\section{Calibration}

The calibration is performed via a standard two-step procedure [29], [30]. First, the power $P_{m}$ backscattered by the built surface, placed at a distance $r_{m}$ from the antenna, is measured to obtain the non-calibrated data

$$
P_{m}=\frac{P_{t} G}{4 \pi r_{m}^{2}} \frac{\sigma_{m}}{4 \pi r_{m}^{2}} K
$$

It depends on the antenna gain $G$, the transmitted power $P_{t}$, and the environmental effect $K$. Then, the surface is replaced by a perfectly conducting trihedral corner reflector, and the received power $P_{t r i}$ is measured at a distance $r_{t r i}$

$$
P_{t r i}=\frac{P_{t} G}{4 \pi r_{t r i}^{2}} \frac{\sigma_{t r r i}}{4 \pi r_{t r i}^{2}} K
$$

Therefore, the calibrated radar cross section $\sigma_{m}$ is obtained by the known trihedral radar cross section $\sigma_{t r i}$ as 

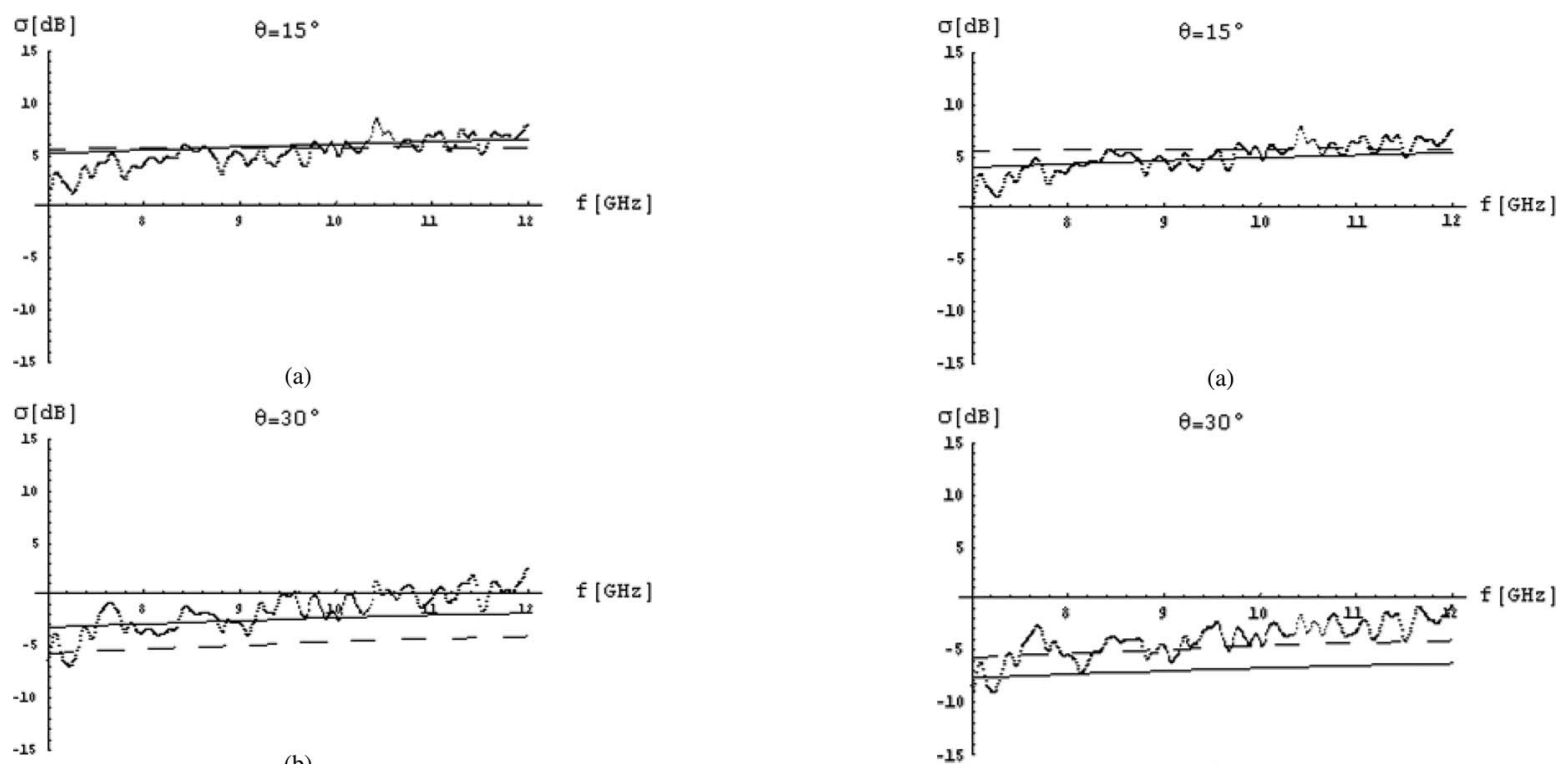

(b)
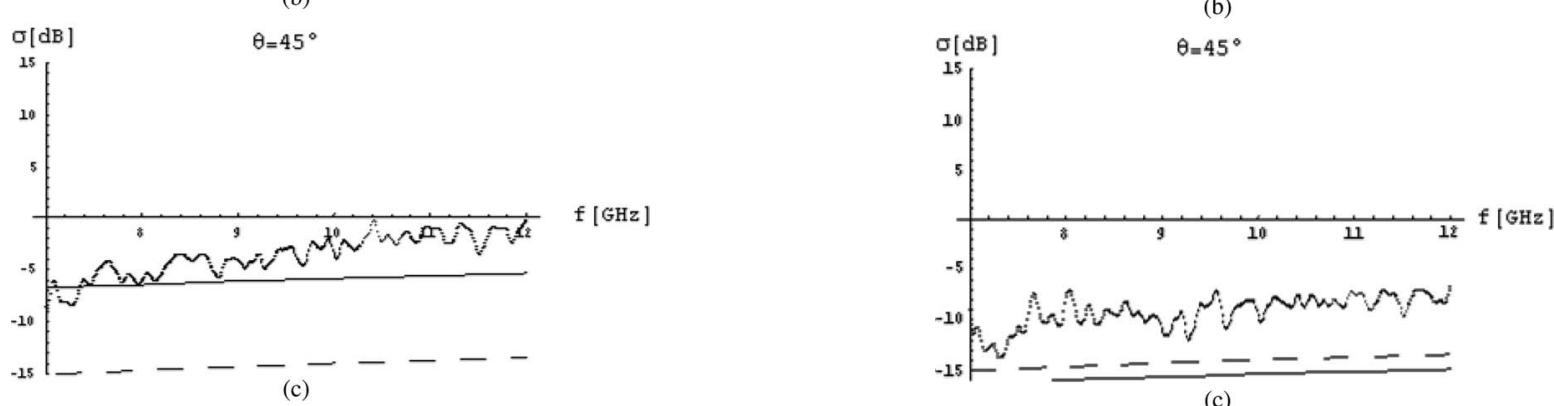

(c)

Fig. 5. VV polarization. Comparison between (dots) data, (dashed line) KA, and (solid line) SPM as a function of the frequency for incidence angle of (a) $15^{\circ}$, (b) $30^{\circ}$, and (c) $45^{\circ}$.

follows:

$$
\sigma_{m}=\frac{r_{m}^{4}}{r_{t r i}^{4}} \frac{P_{m}}{P_{t r i}} \sigma_{t r i}
$$

Based on previous measurement campaigns, the measurement experimental error is expected to be lower than $1 \mathrm{~dB}$ [31]-[33].

\section{RESUlTS}

In this section, we present a comparison between the data measured in accordance with the procedure described in Section IV and the results obtained by employing the theoretical methods recalled in Section III. The set of acquired scattering data allows the investigation of the properties of the backscattered electromagnetic field as a function of the incidence angle and of the electromagnetic frequency.

\section{A. Overall Comparison}

In Fig. 4(a), a comparison between measured data (dots), SPM (solid line), and KA (dashed line) is shown for VV polarization. As stated in Section III, both theoretical methods make use of the $\mathrm{fBm}$ process for the surface description. Each dot is representative of the value averaged over all the $\phi$ angles and the frequency-band measurements. The theoretical curves are also averaged in the considered band. Note that at low incidence angles (up to about $20^{\circ}$ ), KA predictions well match

Fig. 6. HH polarization. Comparison between (dots) data, (dashed line) KA, and (solid line) SPM as a function of the frequency for incidence angle of (a) $15^{\circ}$, (b) $30^{\circ}$, and (c) $45^{\circ}$.

TABLE II VARIANCE AND CORRELATION LENGTH VALUES AS A FUNCTION OF THE OBSERVED AREA

\begin{tabular}{c|c|c}
\hline \hline Area $[\mathrm{cm} 2]$ & $\sigma[\mathrm{cm}]$ & $l[\mathrm{~cm}]$ \\
\hline $90 \times 90$ & 1.39 & 17.3 \\
\hline $50 \times 50$ & 1.3 & 9.1 \\
\hline $30 \times 30$ & 1.01 & 5.9 \\
\hline $10 \times 10$ & 0.7 & 3.3 \\
\hline
\end{tabular}

the experimental data; at larger incidences, we expect that KA validity limits are not satisfied, and the electromagnetic method accuracy decreases. The SPM seems to be able to follow the field behavior for intermediate incidence angles, in accordance with its validity limits [34]. Similar conclusions can be inferred by analysis of Fig. 4(b) for $\mathrm{HH}$ polarization. Anyway, a complete discussion on the method validity calls for a reformulation of limits in terms of fractal parameters.

\section{B. Frequency Dependence}

The field scattered by a surface is strongly dependent on the EM incident wavelength; hence, an accurate study on the dependence of the measured data on the frequency is required in order to verify that the performed averages make sense. Therefore, we investigated the EM scattered field of Fig. 4 as a function of the field frequency for fixed incidence angles, (see Figs. 5 and 6, respectively) for VV and $\mathrm{HH}$ polarizations. 


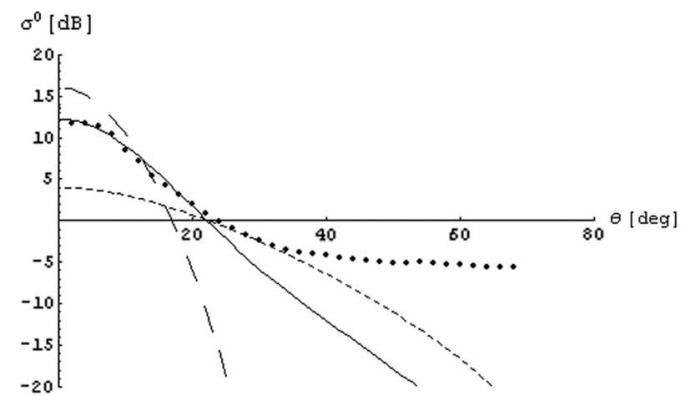

(a)

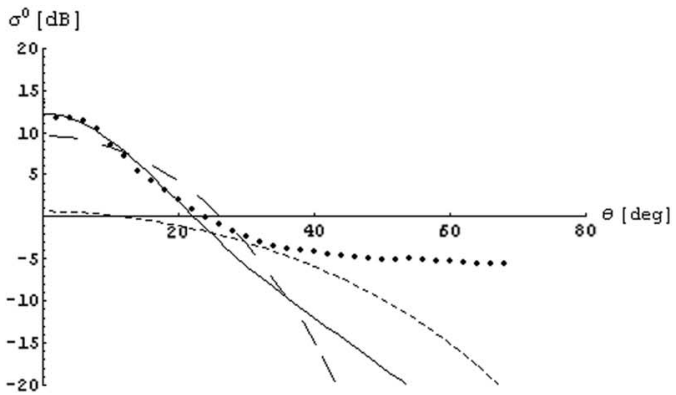

(c)

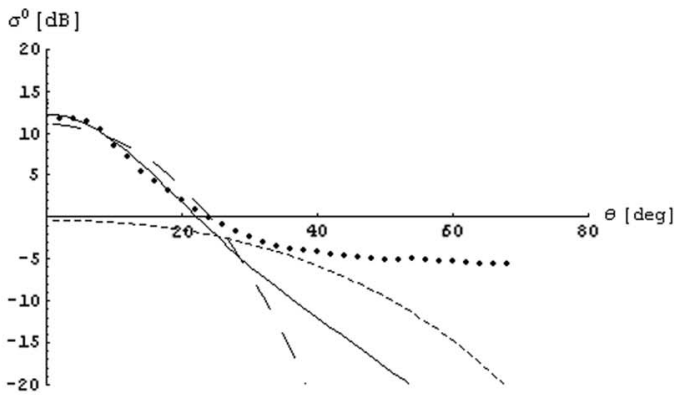

(b)

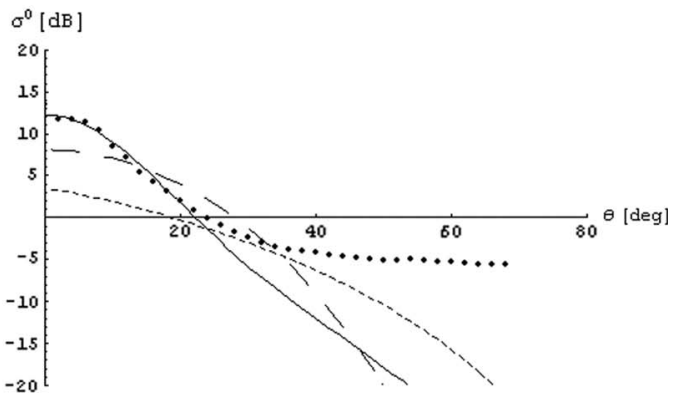

(d)

Fig. 7. VV polarization. Comparison between (dots) experimental results and the KA results in conjunction with (solid line) fBm, (long dashed line) Ga-Ga, and (short dashed line) Ga-Exp for as follows. (a) $\sigma=1.39 \mathrm{~cm} ; l=17.3 \mathrm{~cm}$. (b) $\sigma=1.3 \mathrm{~cm} ; l=9.1 \mathrm{~cm}$. (c) $\sigma=1.01 \mathrm{~cm} ; l=5.9 \mathrm{~cm}$. (d) $\sigma=0.7 \mathrm{~cm}$; $l=3.3 \mathrm{~cm}$

In Figs. 5(a) and 6(a), we show a comparison between measured data (dots), SPM (solid line), and KA (dashed line) as a function of the field frequency, for $\theta$ fixed at $15^{\circ}$. At this incidence angle, there is a good matching between measurement data and prediction provided by both theoretical models, see Fig. 4. Apart from small random oscillations, measured data show a frequency behavior similar to those of the theoretical curves. Such a result confirms that averaging data over the employed frequency range is meaningful for comparative purposes.

In Figs. 5(b) and 6(b), the $\theta$ value is fixed at $30^{\circ}$. We still note that the frequency distribution of the acquired field values is almost linear with a law similar to that of the theoretical method results, again justifying averaging data over the employed frequency range for comparison purposes. For the highest frequencies, we can note a worst agreement between the theoretical models predictions and the experimental data results, due to the fact that the built-surface roughness is scarcely controlled at the smallest spatial scales, which are involved in the scattering at the highest frequencies. In addition, at the highest frequencies, the electromagnetic methods are not completely adequate to model the observed phenomenon, because they do not account for shadow and multiple-reflections phenomena. The reduced frequency band where this behavior occurs does not impair the comparison results.

In Figs. 5(c) and 6(c), the $\theta$ value is fixed at $45^{\circ}$, in correspondence of a significant mean difference between data and experiments, see Fig. 4. Again, the frequency distribution of the acquired field values is almost linear with a law similar to that of the theoretical method results, justifying averaging data over the employed frequency range for comparison purposes.

So far, we limited our attention on SPM and KA based on the $\mathrm{fBm}$ surface description. Despite it is widely accepted that natural surfaces are efficiently described by fractal models, we find it useful to compare fractal and classical methods. The results of such a comparison can drive the choice of the surface model to be employed in remote sensing applications.

\section{Gaussian Versus Fractal Surface Models}

In the following, we compare the experimental data with the results of the KA and SPM electromagnetic methods obtained by employing $\mathrm{fBm}$ and classical surface models, i.e., a Gaussian surface pdf, with Gaussian (Ga-Ga) or exponential (Ga-Exp) correlation function.

In order to perform the comparison, it is necessary to determine the correlation length and the surface height variance. Therefore, we estimated these parameters on the largest possible square area $\left(90 \times 90 \mathrm{~cm}^{2}\right)$ of the built surface: The obtained results are presented in the first row of Table II. In Figs. 7(a) and 8(a), the comparison between the measured scattered fields (dots) and the theoretical results obtained by using fBm (solid line), Ga-Ga (long dashed line) and Ga-Exp (short dashed line) surface models in the KA is provided for $\mathrm{VV}$ and $\mathrm{HH}$ polarizations, respectively. In Figs. 9(a) and 10(a), the same analysis is shown for the SPM method.

It is evident that the fBm surface model better matches the data with respect to classical models, at least for small and moderate incidence angles. The only exception regards the HH polarization for the SPM case. The differences between fractal and classical scattering model results are surprisingly wide, and they lead to think that the used classical parameter values are not representative of the considered surface. Therefore, we evaluated the classical parameter values $l$ and $\sigma$ by considering smaller portions of the built surface (see from the second to the fourth rows of Table II), and we compared the correspondent evaluated EM scattered field with the measured data, as shown in Figs. 7(b)-(d), 8(b)-(d), 9(b)-(d), and 


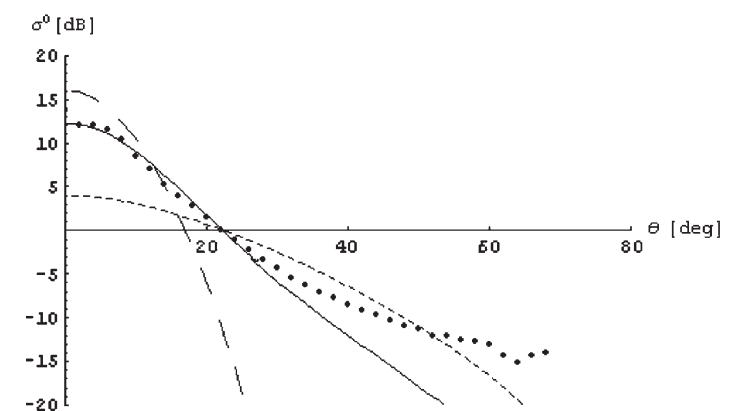

(a)

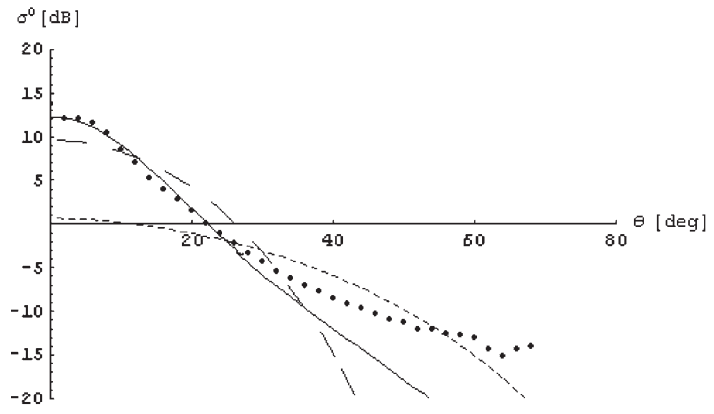

(c)

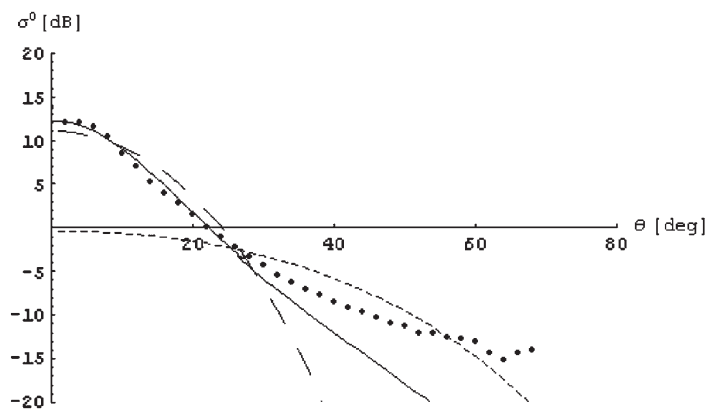

(b)

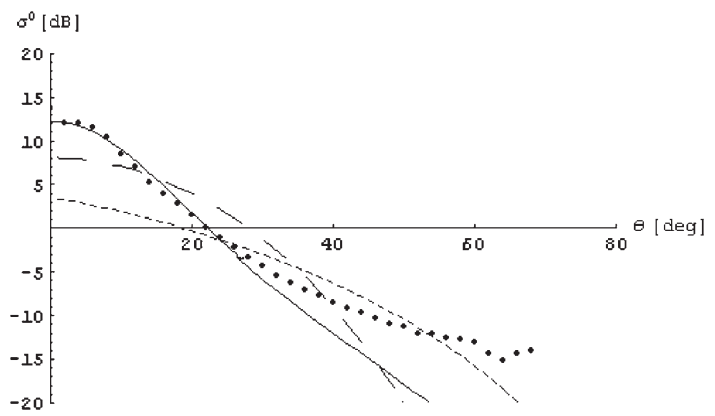

(d)

Fig. 8. HH polarization. Comparison between (dots) experimental results and the KA results in conjunction with (solid line) fBm, (long dashed line) Ga-Ga, and (short dashed line) Ga-Exp for as follows. (a) $\sigma=1.39 \mathrm{~cm} ; l=17.3 \mathrm{~cm}$. (b) $\sigma=1.3 \mathrm{~cm} ; l=9.1 \mathrm{~cm}$. (c) $\sigma=1.01 \mathrm{~cm} ; l=5.9 \mathrm{~cm}$. (d) $\sigma=0.7 \mathrm{~cm}$; $l=3.3 \mathrm{~cm}$.

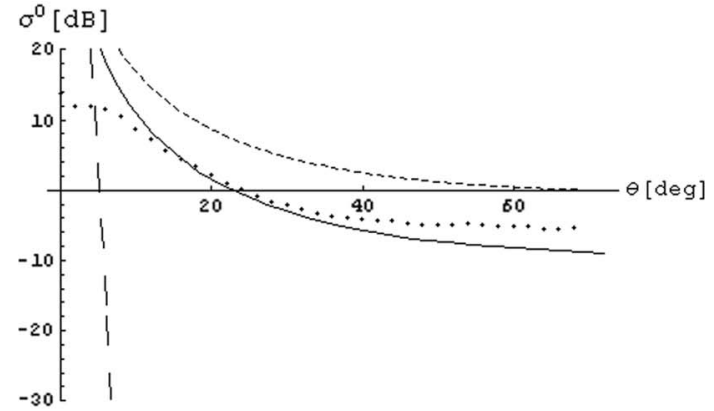

(a)

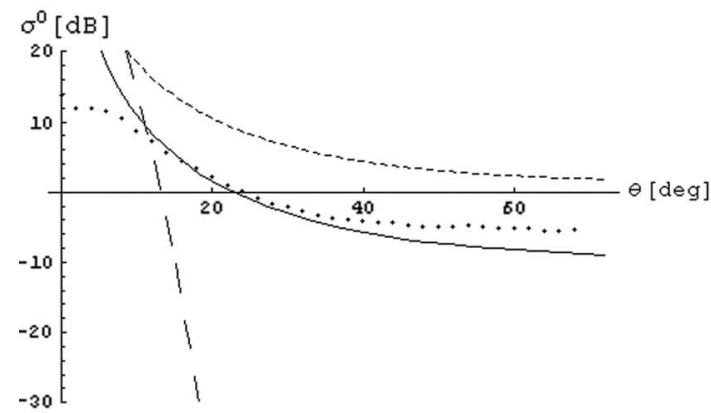

(c)

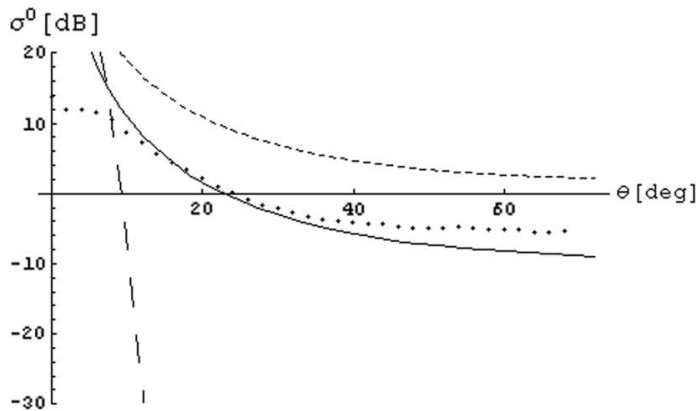

(b)

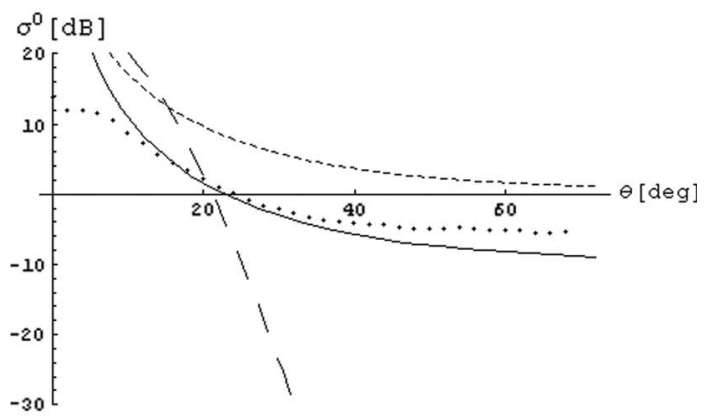

(d)

Fig. 9. VV polarization. Comparison between (dots) experimental results and the SPM results in conjunction with (solid line) fBm, (long dashed line) Ga-Ga, and (short dashed line) Ga-Exp for as follows. (a) $\sigma=1.39 \mathrm{~cm} ; l=17.3 \mathrm{~cm}$. (b) $\sigma=1.3 \mathrm{~cm} ; l=9.1 \mathrm{~cm}$. (c) $\sigma=1.01 \mathrm{~cm} ; l=5.9 \mathrm{~cm}$. (d) $\sigma=0.7 \mathrm{~cm}$; $l=3.3 \mathrm{~cm}$.

10(b)-(d). As expected, for classical surface descriptions, $l$ and $\sigma$ values depend on the dimension of the surface used to evaluate them. Figs. 7-10 show that the variations of the $l$ and $\sigma$ values strongly affect the evaluation of the scattered field. As a dramatic consequence, this strongly reduces reliability of results obtained by employing classical scattering theories and their use for inverse problems in remote sensing applications. Note that we can obtain a reasonable agreement between measurements and data by appropriately choosing the Gaussian parameters $\sigma$ and $l$ : for instance, in the considered case study, the KA with $\mathrm{Ga}-\mathrm{Ga}$ surface model with $\sigma=1.3 \mathrm{~cm}$ and $l=9.1 \mathrm{~cm}$ allows the obtaining of a rather good agreement 


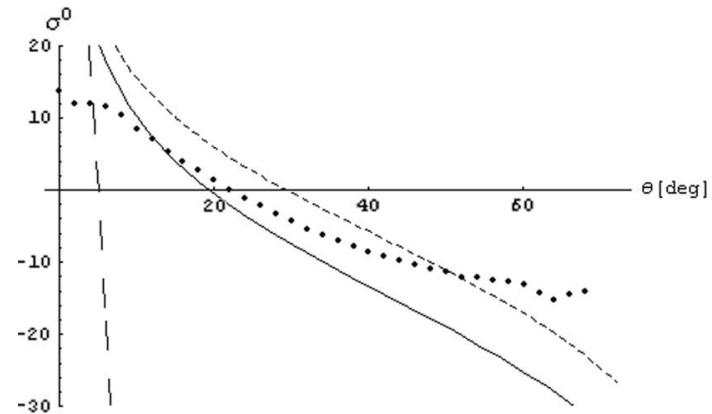

(a)

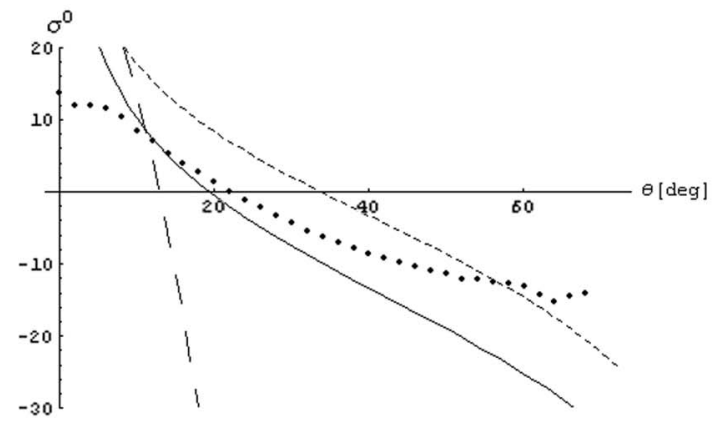

(c)

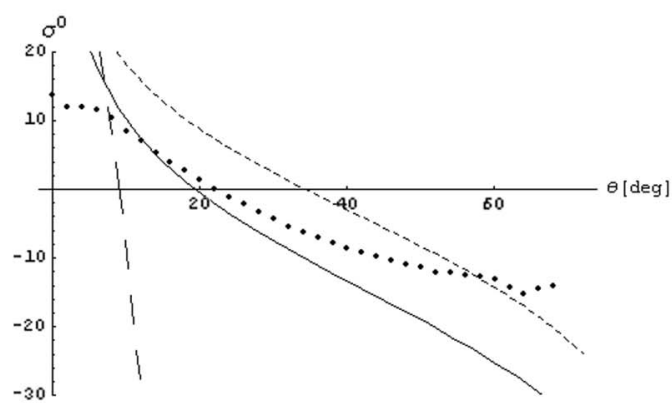

(b)

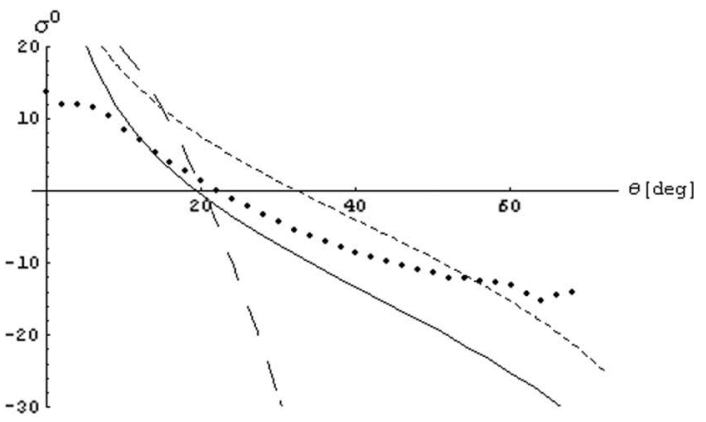

(d)

Fig. 10. HH polarization. Comparison between (dots) experimental results and the SPM results in conjunction with (solid line) fBm, (long dashed line) Ga-Ga, and (short dashed line)Ga-Exp for as follows. (a) $\sigma=1.39 \mathrm{~cm} ; l=17.3 \mathrm{~cm}$. (b) $\sigma=1.3 \mathrm{~cm} ; l=9.1 \mathrm{~cm}$. (c) $\sigma=1.01 \mathrm{~cm} ; l=5.9 \mathrm{~cm}$. (d) $\sigma=0.7 \mathrm{~cm}$; $l=3.3 \mathrm{~cm}$.

with measurements for angles lower than $20^{\circ}$, [see Figs. $7(\mathrm{~b})$ and $8(\mathrm{~b})]$. However, the $\sigma$ and $l$ values that guarantee this agreement are not representative of the surface.

The obtained results lead to the conclusion that fractal models are more appropriate than classical ones, for the following reasons: 1) the electromagnetic results are closer to experimental data, and 2) the fractal parameters better describe the surfaces because they are intrinsic parameters, i.e., their evaluation does not depend on the observer. Both results suggest the use of fractals for the electromagnetic scattering evaluation as well as for inverse remote-sensing problems.

\section{CONCLUSion}

In this paper, we presented an innovative measurement procedure for validating the theoretical methods of evaluation of the EM field scattered from natural surfaces. To this aim, a fractal surface with assigned parameters was built as a superposition of cardboard and aluminum layers, as presented in a recent paper. The characteristics of such a surface were verified by measuring it with an optical high-precision instrument. The surface was mounted on a rotor in an anechoic chamber, and the EM field backscattered from it was measured at different incidence angles. Problems related with the acquisition of the field values were addressed and solved.

The comparison between the obtained calibrated data and the theoretical results deriving from the $\mathrm{fBm}$ use in the KA and SPM methods shows matching and discrepancies between theoretical prediction and experimental results. In particular, at low incidence angles, the KA appears to be the most appropriate method to predict the scattered field, whereas at intermediate angles, the SPM better matches the data, as predicted by theory.

In addition, provided that the natural surfaces are well described by fractal laws, we explored the possibility that classical methods relying on the Gaussian surface description could be used for the evaluation of the field scattered from natural surfaces. Therefore, we measured the standard deviation $\sigma$ and the correlation length $l$ on surface portions with different sizes, and we compared the predicted EM scattered field with data. The obtained $\sigma$ and $l$ values and, as a consequence, the forecasted scattered field values turn out to depend on the chosen dimension. It means that the classical $\sigma$ and $l$ parameters are not intrinsic descriptors of the surface. The obtained experimental results lead to the conclusion that the use of fractal surfaces in the EM scattering theories provides two main advantages: 1) the results are closer to experimental data, and 2) the surface is efficiently described in terms of only two intrinsic parameters, whose value does not depend on the observer.

\section{REFERENCES}

[1] G. Franceschetti and D. Riccio, Scattering, Natural Surfaces, and Fractals. Burlington, MA: Academic, 2007.

[2] G. Franceschetti, A. Iodice, and D. Riccio, "Fractal models for scattering from natural surfaces," in Scattering, R. Pike and P. Sabatier, Eds. London, U.K.: Academic, 2001, pp. 467-485.

[3] T. K. Chan, Y. Kuga, A. Ishimaru, and C. T. C. Le, "Experimental studies of bistatic scattering from two-dimensional conducting random rough surfaces," IEEE Trans. Geosci. Remote Sens., vol. 34, no. 3, pp. 674-680, May 1996.

[4] Y. Oh, K. Sarabandi, and F. T. Ulaby, "An empirical model and an inversion technique for radar scattering from bare soil surfaces," IEEE Trans. Geosci. Remote Sens., vol. 30, no. 2, pp. 370-381, Mar. 1992.

[5] K. A. O'Donnell and E. R. Mendez, "Experimental study of scattering from characterized random surfaces," J. Opt. Soc. Amer. A, Opt. Image Sci., vol. 4, no. 7, pp. 1194-1205, Jul. 1987.

[6] P. Beckmann, "Scattering by non-Gaussian surfaces," IEEE Trans. Antennas Propag., vol. AP-21, no. 2, pp. 169-175, Mar. 1973.

[7] M. Davidson, T. Le Toan, F. Mattia, G. Satalino, T. Manninen, and M. Borgeaud, "On the characterization of agricultural soil roughness for radar remote sensing studies," IEEE Trans. Geosci. Remote Sens., vol. 38 , no. 2 , pp. 630-640, Mar. 2000. 
[8] S. C. Wu, M. F. Chen, and A. K. Fung, "Non-Gaussian surface generation," IEEE Trans. Geosci. Remote Sens., vol. 26, no. 6, pp. 885-888, Nov. 1988

[9] B. B. Mandelbrot, The Fractal Geometry of Nature. New York: Freeman, 1983.

[10] K. Falconer, Fractal Geometry: Mathematical Foundations and Applications. New York: Wiley, 1990.

[11] J. S. Feder, Fractals (Physics of Solids and Liquids). New York: Plenum, 1988.

[12] B. B. Mandelbrot and V. Ness, "Fractional Brownian motions, fractional noises and applications," SIAM Rev., vol. 10, no. 4, pp. 422-437, Oct. 1968.

[13] P. Flandrin, "On the spectrum of fractional Brownian motions," IEEE Trans. Inf. Theory, vol. 35, no. 1, pp. 197-199, Jan. 1989.

[14] H. Maitre and M. Pinciroli, "Fractal characterization of a hydrological basin using SAR satellite images," IEEE Trans. Geosci. Remote Sens., vol. 37, no. 1, pp. 175-181, Jan. 1999.

[15] J. E. Summers, R. J. Soukup, and R. F. Gragg, "Mathematical modeling and computer-aided manufacturing of rough surfaces for experimental study of seafloor scattering," IEEE J. Ocean. Eng., vol. 32, no. 4, pp. 897914, Oct. 2007.

[16] C. Y. Poont, R. S. Saylest, and T. A. Jones, "Surface measurement and fractal characterization of naturally fractured rocks," J. Phys. D, Appl. Phys., vol. 25, no. 8, pp. 1269-1275, Aug. 1992.

[17] D. L. Turcotte, "The relationship of fractals in geophysics to "the new science"," Chaos Solitons Fractals, vol. 19, no. 2, pp. 255-258, Jan. 2004

[18] G. Franceschetti, A. Iodice, S. Maddaluno, and D. Riccio, "A fractalbased theoretical framework for the retrieval of surface parameters from electromagnetic backscattering data," IEEE Trans. Geosci. Remote Sens., vol. 38, no. 2, pp. 641-650, Mar. 2000.

[19] E. Ceraldi, G. Franceschetti, A. Iodice, and D. Riccio, "Estimating the soil dielectric constant via scattering measurements along the specular direction," IEEE Trans. Geosci. Remote Sens., vol. 43, no. 2, pp. 295305, Feb. 2005.

[20] G. Ruello, P. Blanco, A. Iodice, J. J. Mallorqui, D. Riccio, A. Broquetas, and G. Franceschetti, "Synthesis, construction and validation of a fractal surface," IEEE Trans. Geosci. Remote Sens., vol. 44, no. 6, pp. 14031412, Jun. 2006.

[21] G. Franceschetti, A. Iodice, M. Migliaccio, and D. Riccio, "Scattering from natural rough surfaces modeled by fractional Brownian motion twodimensional processes," IEEE Trans. Antennas Propag., vol. 47, no. 9, pp. 1405-1415, Sep. 1999.

[22] G. Franceschetti, A. Iodice, M. Migliaccio, and D. Riccio, "Fractals and the small perturbation scattering model," Radio Sci., vol. 34, no. 5, pp. 1043-1054, Sep./Oct. 1999.

[23] G. Franceschetti, M. Migliaccio, D. Riccio, and G. Schirinzi, "SARAS: A SAR raw signal simulator," IEEE Trans. Geosci. Remote Sens., vol. 30, no. 1, pp. 110-123, Jan. 1992.

[24] S. R. Brown and C. H. Sholz, "Broad band study of the topography of natural rock surfaces," J. Geophys. Res., vol. 90, no. B14, pp. 1257512582, Dec. 1985.

[25] W. M. Krueger, S. D. Jost, and K. Rossi, "On synthesizing discrete fractional Brownian motion with applications to image processing," Graph. Models Image Process., vol. 58, no. 4, pp. 334-344, Jul. 1996.

[26] F. W. Millet and K. F. Warnick, "Validity of rough surface backscattering models," Waves Random Media, vol. 14, no. 3, pp. 327-347, Jul. 2004

[27] H. A. Zebker and J. Villasenor, "Decorrelation in interferometric radar echoes," IEEE Trans. Geosci. Remote Sens., vol. 30, no. 5, pp. 950-959, Sep. 1992.

[28] F. K. Li and R. M. Goldstein, "Studies of multibaseline spaceborne interferometric synthetic aperture radars," IEEE Trans. Geosci. Remote Sens., vol. 28, no. 1, pp. 88-97, Jan. 1990.

[29] B. M. Kent, "Comparative measurements of precision radar cross section (RCS) calibration targets," in Proc. Antennas Propag. Symp., 2001, vol. 4, pp. $412-415$.

[30] J. M. Geffrin, P. Sabouroux, and C. Eyraud, "Free space experimental scattering database continuation: Experimental set-up and measurement precision," Inv. Probl., vol. 21, no. 6, pp. S117-S130, Nov. 2005.

[31] L. Jofre, "Research and new developments in radar cross section prediction and measurement in Spain," in Proc. 23rd Eur. Microw. Conf. Oct. 1993, pp. 42-46.

[32] S. Pivnenko, J. E. Pallesen, O. Breinbjerg, M. Sierra, P. Caballero, C. Martínez-Portas, J. L. Besada, J. Romeu, S. Blanch, J. M. Gonzalez.Arbesú, C. Sabatier, A. Calderone, G. Portier, H. Eriksson, and J. Zackrisson, "Comparison of antenna measurement facilities with the DTU-ESA $12 \mathrm{GHz}$ validation standard antenna within the EU antenna centre of excellence," IEEE Trans. Antennas Propag., vol. 57 , no. 7, pp. 1863-1878, Jul. 2009.

[33] M. Martin-Neira, I. Cabeza, C. Perez, M. A. Palacios, M. A. Guijarro, S. Ribo, I. Corbella, S. Blanch, F. Torres, N. Duffo, V. Gonzalez, S. Beraza, A. Camps, M. Vall-llossera, S. Tauriainen, J. Pihlflyckt, J. P. Gonzalez, and F. Martin-Porqueras, "AMIRAS—An airborne MIRAS demonstrator," IEEE Trans. Geosci. Remote Sens., vol. 46, no. 3, pp. 705716, Mar. 2008.

[34] F. T. Ulaby, R. K. Moore, and A. K. Fung, Microwave Remote Sensing: Active and Passive. Reading, MA: Addison-Wesley, 1982.

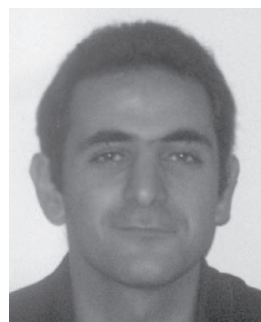

Giuseppe Ruello (S'00-M'04) was born in Naples, Italy, on February 12, 1975. He received the Laurea degree (with honors) in telecommunication engineering and the Ph.D. degree in information engineering from the University of Naples "Federico II," Naples, in 1999 and 2003, respectively.

In 2000, he received a grant from the University of Naples to be spent at the Department of Electronic and Telecommunication Engineering for research in the field of remote sensing. In 2000, he was also the recipient of a grant from the University of Rome "La Sapienza," Rome, Italy. In 2002, as well as in 2004-2005, he was a Visiting Scientist in the Department of Signal Theory and Communications, Universitat Politecnica de Catalunya, Barcelona, Spain. He is currently a Research Scientist with the Department of Electronic and Telecommunication Engineering, University of Naples. He is the author of about 150 papers published in refereed journals or in proceedings of international and national conferences. His main research interests are in the field of SAR remote sensing, modeling of electromagnetic scattering from natural surfaces, SAR raw-signal simulation, modeling of electromagnetic field propagation in urban environment, and remote-sensing techniques for developing countries.

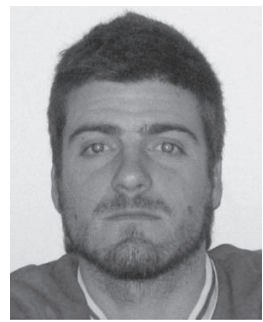

Pablo Blanco-Sánchez was born in Terrassa, Spain, in 1977. He received the B.Sc. degree in physics from Valencia University, Valencia, Spain, in 2001. $\mathrm{He}$ is currently working toward the Ph.D. degree in the Department of Signal Theory and Communications, School of Telecommunication Engineering, Universitat Politècnica de Catalunya, Barcelona, Spain.

From September to December 2004, he was with the Istituto per il Rilevamento Elettromagnetico dell'Ambiente, Naples, Italy, in the frame of his Ph.D. work. In 2007, he joined the Remote Sensing Department, Institut Cartogràfic de Catalunya, Barcelona. His main research interests are in the areas of synthetic aperture radar (SAR) interferometry and SAR differential interferometry.

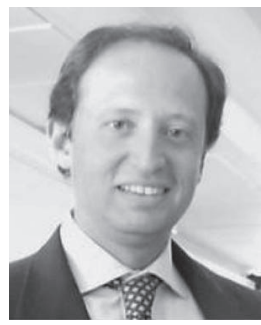

Antonio Iodice (S'97-M'00-SM'04) was born in Naples, Italy, on July 4, 1968. He received the Laurea degree (cum laude) and the Ph.D. degree in electronic engineering and computer science from the University of Naples "Federico II," Naples, in 1993 and 1999, respectively.

In 1995, he received a grant from the Italian National Council of Research to be spent at the Istituto di Ricerca per l'Elettromagnetismo e i Componenti Elettronici, Naples, for research in the field of remote sensing. He was with Telespazio S.p.A., Rome, Italy, from 1999 to 2000. Since 2000, he has been with the Department of Electronic and Telecommunication Engineering, University of Naples "Federico II," where he is currently a Professor of electromagnetics. He is the author or coauthor of about 150 papers published in refereed journals or in proceedings of international and national conferences. His main research interests are in the field of microwave remote sensing and electromagnetics: modeling of electromagnetic scattering from natural surfaces and urban areas, simulation and processing of synthetic aperture radar (SAR) signals, SAR interferometry, and electromagnetic propagation in urban areas.

Dr. Iodice was the recipient of the 2009 Sergei A. Schelkunoff Prize Paper Award from the IEEE Antennas and Propagation Society. 


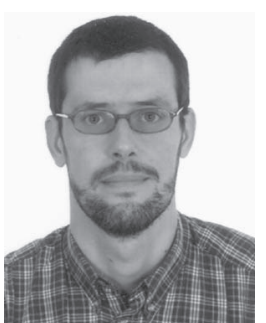

Jordi J. Mallorquí (S'93-M'96) was born in Tarragona, Spain, in 1966. He received the Ingeniero degree in telecommunications engineering and the Dr. Ing. degree in telecommunications engineering for his research on microwave tomography for biomedical applications from the Universitat Politècnica de Catalunya (UPC), Barcelona, Spain, in 1990 and 1995 , respectively.

Since 1993, he has been teaching with the Department of Signal Theory and Communications, School of Telecommunications Engineering, UPC, where he was first an Assistant Professor and, then, since 1997, has been an Associate Professor. His teaching activity involves microwaves, radionavigation systems, and remote sensing. He spent a sabbatical year with the Jet Propulsion Laboratory, Pasadena, CA, in 1999, where he worked on interferometric airborne synthetic aperture radar (SAR) calibration algorithms. He is currently working on the application of SAR interferometry to terrain-deformation monitoring with orbital, airborne, and ground data; vessel detection and classification from SAR images; and 3-D electromagnetic (EM) simulation of SAR systems. He is also collaborating in the design and construction of a ground-based SAR interferometer for landslide control. Finally, he is currently developing the hardware and software of a bistatic opportunistic SAR for interferometric applications using ERS, ENVISAT, and TerraSAR-X as sensors of opportunity. He has published more than 80 papers on microwave tomography, EM numerical simulation, and SAR processing, interferometry, and differential interferometry in refereed journals and international symposia.

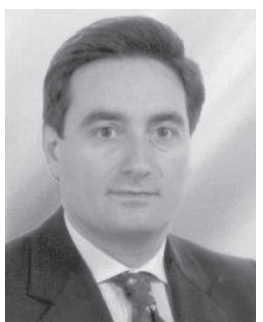

Daniele Riccio (M'91-SM'99) was born in Naples, Italy, on April 13, 1962. He received the Laurea degree (cum laude) in electronic engineering from the University of Naples "Federico II," Naples, in 1989.

He was a Research Scientist with the Institute of Research on Electromagnetics and Electronic Components, Italian National Council of Research (CNR), and at the Department of Biomedics, Electronic and Telecommunication Engineering, University of Naples. He was also a Guest Scientist at the High-Frequency Institute, German Aerospace Center, Munich, Germany, in 1994 and 1995, and a Visiting Professor at the Universitat Politècnica de Catalunya, Barcelona, Spain, in 2006. He is currently a Professor of electromagnetics and remote sensing with the Department of Electronic and Telecommunication Engineering, University of Naples. His research activities have been published in three books and more than 200 published papers. His research interests are mainly focused on microwave remote sensing, synthetic aperture radar with emphasis on data simulation modeling, and information retrieval for land, oceanic, and urban scenes, as well as on the application of fractal geometry to remote sensing and electromagnetic scattering from natural surfaces. He is an Associate Editor for the journals Remote Sensing, Sensors, and The Open Remote Sensing Journal.

Prof. Riccio was the recipient of the 2009 Sergei A. Schelkunoff Prize Paper Award for the best paper published in 2008 on the IEEE TRANSACTIONS ON ANTENnas AND PROPAGation. He was also the recipient of several fellowships from private and public companies (SIP, Selenia, CNR, CORISTA, CRATI) for research in the remote sensing field.

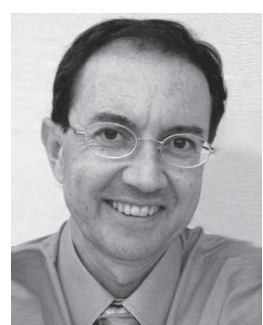

Antoni Broquetas (S'84-M'90) was born in Barcelona, Spain, in 1959. He received the Ingeniero degree in telecommunication engineering and the Dr. Ing. degree in telecommunications engineering for his work on microwave tomography from the Universitat Politècnica de Catalunya (UPC), Barcelona, in 1985 and 1989, respectively.

In 1986, he was a Research Assistant with the Portsmouth Polytechnic, Portsmouth, U.K., where he was involved in propagation studies. In 1987, he joined the Department of Signal Theory and Communications, School of Telecommunication Engineering, UPC, where since 1999, he has been a Full Professor involved in research on radar imaging and remote sensing and, since 2003, has been the Director. From 1998 to 2002, he was the Subdirector of Research with the Institute of Geomatics, Barcelona. He has published more than 150 papers on microwave tomography, radar, ISAR and SAR systems, SAR processing, and interferometry.

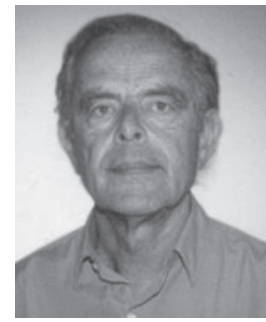

Giorgio Franceschetti (S'60-M'62-SM' 85F'88-LF'01) was born and educated in Italy.

He was a Distinguished Visiting Scientist with the Jet Propulsion Laboratory, Pasadena, CA, and a Visiting Professor with the University of Illinois at Urbana-Champaign, in 1976 and 1977; with the University of California at Los Angeles, Los Angeles, in 1981 and 1983; with the National Somali University, Mogadishu, Somalia, in 1984; and with the University of Santiago de Compostela, Santiago de Compostela, Spain, in 1995. Since 1968, he has been with the Department of Electronic and Telecommunication Engineering, University of Naples "Federico II," Naples, Italy, where he is currently a Full Professor of electromagnetic wave theory. He is the author of over 170 papers in peer-reviewed international scientific journals and 10 books.

Prof. Franceschetti was the recipient of a large number of domestic and international awards, the last one being the gold medal from the President of Italy (2001), Distinguished Accomplishment Award from the IEEE GRS Society, and Schelkunoff Prize (for the second time) from the IEEE Antennas and Propagation Society (1999 and 2008). 